\title{
Analyse comparée des effets de dragage et de chasse hydraulique sur l'envasement à l'amont du bassin maritime de la Rance
}

\author{
Chantal Bonnot-Courtois \\ Laboratoire de Géomorphologie E.P.H.E. \\ URA 904 du CNRS "Dynamique et gestion des espaces littoraux » \\ 15 boulevard de la mer, 35800 Dinard
}

\section{Introduction}

L'usine marémotrice de la Rance implantée dans un secteur de très grandes marées, utilise un bassin de retenue de 180 millions de $\mathrm{m}^{3}$ d'eau et n'a pas connu de problèmes de sédimentation très importants depuis sa mise en service en 1967. Par contre, depuis 1989, la sédimentation vaseuse s'est accélérée dans la partie amont du bassin maritime, nécessitant des dragages d'entretien du chenal de navigation pendant plusieurs années consécutives. Cette étude concerne d'une part, le devenir des rejets de dragage afin de suivre l'extension du panache turbide et d'autre part, la dynamique des matières en suspension dans cette partie de l'estuaire. Seront ensuite analysées les causes de l'accélération de l'envasement et des tentatives de solution avec le suivi d'une chasse hydraulique expérimentale réalisée en 1993.

\section{Présentation du bassin maritime de la Rance}

La partie terminale de la vallée de la Rance débouche dans le golfe normand-breton par une ria très découpée dans la série métamorphique briovérienne du massif de SaintMalo. La découpure de la ria est largement guidée par la géologie, avec une succession de bandes orientées SW-NE qui comprennent du Sud au Nord des granulites, les schistes et phyllades de Saint-Lô, des schistes, micaschistes et gneiss, puis de nouveau les granulites feuilletées du massif de Saint-Malo (B.R.G.M., 1964). La morphologie de la ria dépend de la résistance des roches qu'elle entaille, les anses larges correspondant aux schistes et micaschistes et les seuils rocheux étroits étant encadrés par des falaises élevées de gneiss et de granulites plus résistants (CHOUx, 1959).

Le bassin maritime proprement dit s'étend sur une longueur d'une vingtaine de $\mathrm{km}$ entre l'usine marémotrice au Nord et l'écluse du Chatelier au Sud qui marque la limite amont de l'incursion des eaux marines (fig. 1), et correspond à une surface du bassin de retenue de $22 \mathrm{~km}^{2}$. Le débit de la Rance fluviale est en moyenne de $7 \mathrm{~m}^{3} / \mathrm{s}$, avec un débit d'étiage de $0,5 \mathrm{~m}^{3} / \mathrm{s}$ et une crue décennale de $80 \mathrm{~m}^{3} / \mathrm{s}$. Ces valeurs sont extrêmement faibles par rapport au volume oscillant de la marée qui représente des débits entrants au niveau de l'usine marémotrice de l'ordre de $9000 \mathrm{~m}^{3} / \mathrm{s}$ en morte-eau et de $18000 \mathrm{~m}^{3} / \mathrm{s}$ en vive-eau. Cette prépondérance de l'action de la marée par rapport au débit fluviatile se traduit dans la répartition de la salinité qui subdivise l'estuaire en 3 parties du Nord au Sud (fig. l):

\section{Comparative study of dredging and flushing effects \\ on sedimentation in the upper part of the Rance estuary}

Since a few years, an exceptional muddy sedimentation increase was observed in the upper part of the Rance estuary, and a dredging of the navigation channel was required. But the dredged sediments were transported at a short distance only because ebb current is too weak to allow the discharge of suspended load in the lower part of the maritime basin. The excess of fine particles deposition is linked to drought and a low river inflow during three years in a row. A fluvial basin located at the upper part of the marine stretch of the estuary can be used for hydraulic flushing which happens to be more efficient for fluid mud resuspension and translation of the turbidity maximum towards the outer part of the Rance estuary. 


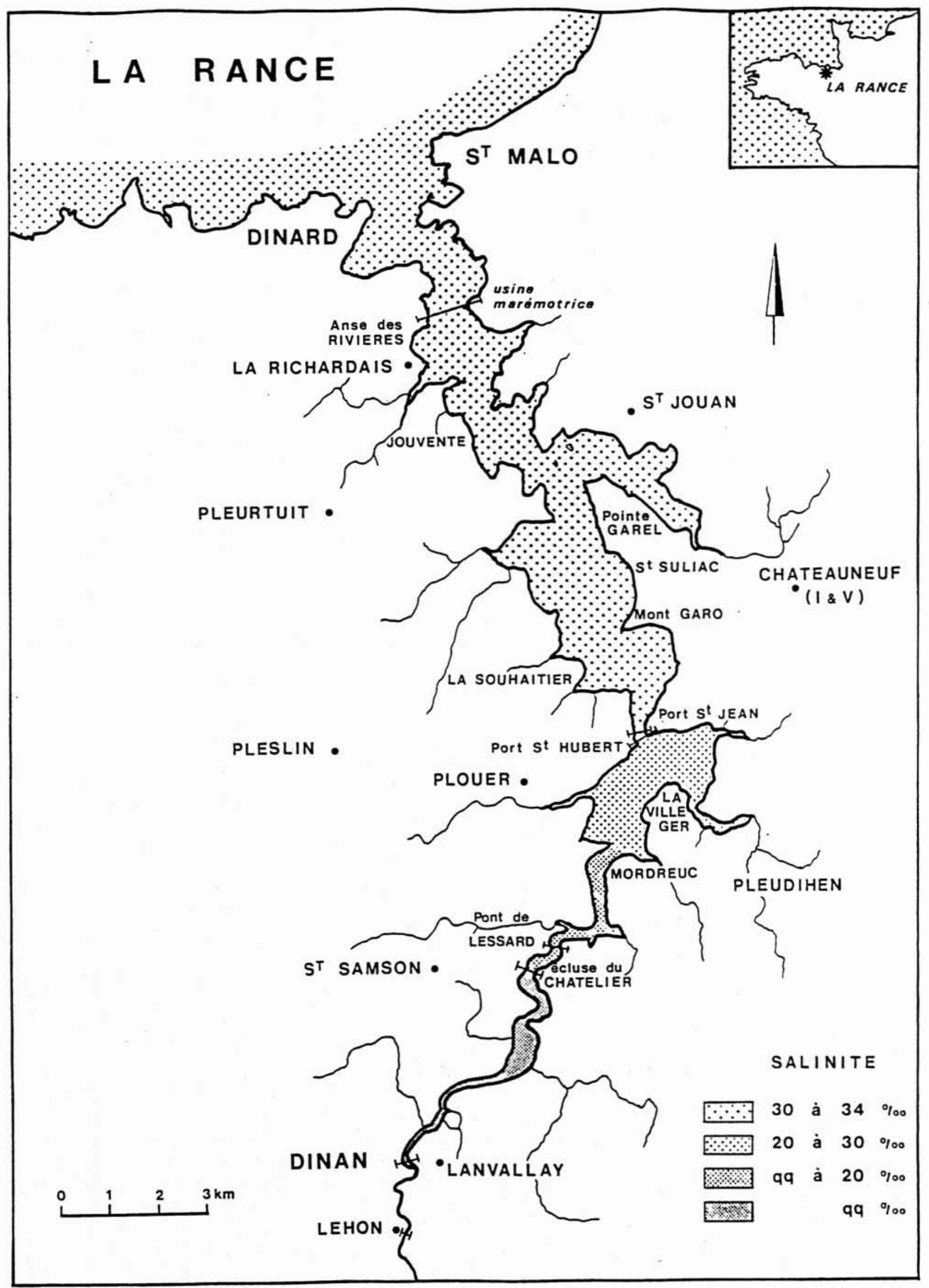

1. Morphologie de la ria et répartition de la salinité dans l'estuaire de la Rance. 
- de l'usine marémotrice à l'étroiture du Port Saint-Jean, la salinité est comprise entre 30 et $34 \%$ et donc très proche de celle de l'eau de mer;

- du Port Saint-Jean à Mordreuc où se resserre la vallée, la salinité reste élevée et varie de 20 à $30 \%$;

- enfin, de Mordreuc à l'écluse du Chatelier, dans la partie très étroite et amont de l'estuaire, on rencontre des eaux douces ou saumâtres selon le moment de la marée et l'épaisseur de la tranche d'eau;

- au-delà de l'écluse du Chatelier jusqu'à Dinan, il s'agit de la Rance fluviale et les eaux sont douces, avec parfois quelques incursions d'eaux marines dans le bassin de Lyvet situé à l'amont immédiat de l'écluse du Chatelier.

La turbidité des eaux de la Rance, aussi bien fluviale que maritime, est mal connue. D'après RoA Morales (1956), les teneurs en matières en suspension dans la Rance sont très faibles par rapport à celles d'autres estuaires. Les valeurs trouvées varient entre 100 et $300 \mathrm{mg} / 1$ pour la partie estuarienne comprise entre Le Chatelier et Mordreuc, et ne dépassent pas $8 \mathrm{mg} / \mathrm{l}$ à l'aval de Port Saint-Jean. En 1982, le L.C.H.F. donne des valeurs de turbidité comprises entre 4 et $8 \mathrm{mg} / \mathrm{l}$ dans la zone du barrage. De plus, la répartition des troubles en suspension dans le golfe normand-breton, analysée grâce à une image LANDSAT (FrALIT, 1979), montre que si les eaux de la côte Ouest du Cotentin et de la baie du Mont Saint-Michel sont très chargées en matières en suspension, au contraire, à l'Ouest de la pointe du Grouin de Cancale, la turbidité des eaux du golfe de Saint-Malo est faible et les eaux de la Rance apparaissent particulièrement peu chargées en matières en suspension.

Etudiée bien avant l'implantation de l'usine marémotrice (Berthois L. et C., 1954, 1955; RoA Morales, 1956; Bourcart et Roa Morales, 1957), la sédimentation de la Rance maritime est caractéristique des estuaires. Les différents types de sédiments se répartissent en fonction des conditions hydrodynamiques. Dans les secteurs d'annulation des courants, au fond des anses latérales de la ria et à l'amont de l'estuaire, se développe une sédimentation fine qui donne naissance à la slikke parfois recouverte de végétation halophile dans les parties les plus hautes des rives. L'envasement des berges de la Rance correspond donc aux lois de la dynamique sédimentaire estuarienne et des dépôts de vase étaient déjà signalés dans les anses sur la carte du Service hydrographique de 1883 (L.C.H.F., 1982).

Près du chenal se trouvent les faciès sableux dont la granulométrie dépend de l'intensité des courants. Dans la partie amont, au niveau de la plaine de Mordreuc, les courants de marée atténués ne peuvent transporter que des sables fins à moyens. En aval de l'estuaire, les sables plus grossiers se retrouvent au niveau des bancs, et les cailloutis occupent le fond du chenal principal dans les zones d'étranglement où les courants sont violents.

L'origine des sédiments de la Rance maritime est multiple et décrite comme un mélange :

- de cailloutis et de sables grossiers, provenant d'apports continentaux et du lessivage des dépôts périglaciaires quaternaires ;
- de sables moyens, issus du remaniement de dépôts transgressifs marins tyrrhéniens (100 000 ans BP), et flandriens (10 000 ans BP) :

- et de vases ayant pour origine le lessivage des limons quaternaires ou des apports marins récents (BERTHOIS L. et C., 1954 ; Bourcart et Roa Morales, 1957 ; Choux, 1959).

L'exploitation de l'usine marémotrice implique des modifications du cycle naturel des marées. Tout d'abord, l'amplitude de la marée a été réduite d'environ $40 \%$, le marnage n'est plus que de 7 à $8 \mathrm{~m}$ en vive-eau et de $2,5 \mathrm{~m}$ en morte-eau (L.C.H.F., 1982). Le niveau du bassin de retenue ne descend que très exceptionnellement au-dessous de $4 \mathrm{~m} \mathrm{CM}$. Cette réduction du marnage entraîne une diminution de la surface du domaine intertidal qui représentait $70 \%$ de la surface totale du bassin en marée de vive-eau (RETIẺRE, 1989). La zone découvrante ne représente plus actuellement que $50 \%$ de la surface totale du bassin. Les surfaces exondées ont peu varié entre Le Chatelier et Port Saint-Jean, mais ont beaucoup diminué entre Port Saint-Jean et le bras de Chateauneuf où les fonds sont en majorité en dessous de $5 \mathrm{~m}$ (L.C.H.F., 1982).

La durée des étales de pleine mer et de basse mer est très prolongée dans le bassin par rapport à la mer ouverte. Les étales durent jusqu'à presque deux heures à l'intérieur du bassin alors qu'elles n'excèdent pas une quinzaine de minutes pour la marée naturelle. Ceci entraîne une modification des courants qui sont faibles à nuls pendant un temps prolongé, et influence directement la dynamique des matières en suspension qui circulent dans l'estuaire. La prolongation des étales laisse aux particules en suspension le temps d'atteindre les fonds et d'y sédimenter, particulièrement dans les zones abritées des anses et dans les secteurs calmes de l'amont.

En 1986, une étude du L.C.H.F. sur l'évolution des conditions de navigation dans la partie amont de l'estuaire de la Rance conclut que "le déplacement des chenaux entraîne des érosions ou des sédimentations de fonds en bordure du chenal entre Plouer et Mordreuc. Entre 1957 et 1963, la largeur du plan d'eau, le tracé du chenal et sa profondeur ont peu évolué. L'envasement aux abords de Mordreuc n'a pas modifié les conditions d'accès dans le chenal de navigation de manière significative. Cela est également vrai pour la zone comprise entre Mordreuc et l'écluse du Chatelier où le chenal est quasiment stable» (L.C.H.F., 1986).

Cependant, depuis 1989, l'envasement du chenal de navigation dans la partie amont de l'estuaire a été tel que des dragages d'entretien ont été nécessaires à proximité de l'écluse du Chatelier, pendant trois années consécutives, en 1990, 1991 et 1992. Les quantités draguées ont été de $6000 \mathrm{~m}^{3}$ en 1990 et de $20000 \mathrm{~m}^{3}$ les années suivantes. En 1991, un suivi des rejets de dragage a pu être effectué afin de caractériser la dynamique des matières en suspension dans l'estuaire et de suivre l'efficacité du dragage (BONNOTCourtors et LAFOND, 1991). Ces études ont permis de rechercher les causes de l'accélération de l'envasement depuis 1989 et de proposer des solutions visant à utiliser les débits fluviatils pour évacuer les sédiments vers l'aval. 
L'efficacité d'une chasse hydraulique expérimentale a pu être testée en 1993 et les conditions optimales de chasse ont ainsi pu être déterminées.

\section{Suivi de dragage}

\subsection{Conditions Expérimentales}

Compte tenu de l'étroitesse du chenal, la drague de faible puissance était positionnée près du musoir de l'écluse du Chatelier (Photo ${ }^{\circ} 1$ ) et l'extrémité du tuyau, théoriquement attaché à l'une des perches qui borde le chenal, était située en 1991 à presque $1 \mathrm{~km}$ en aval de l'écluse (fig. 2). Les rejets de dragage ont lieu pendant la vidange du bassin, et débutent une heure après la pleine mer pour stopper une à deux heures avant la basse mer, selon le niveau, la drague s'échouant pour des niveaux du bassin inférieurs à $9 \mathrm{~m}$ (fig. 2).

Les prélèvements d'eau ont été effectués en plusieurs points répartis régulièrement entre le point de rejet à l'amont et le Port Saint-Jean à l'aval (fig. 2). Les distances linéaires qui séparent les points de prélèvements de l'écluse du Chatelier sont respectivement d'amont en aval :

- $1 \mathrm{~km}$ pour le point situé en aval du pont de Lessard,

- $2 \mathrm{~km}$ au niveau de Quincoubre,

- 3,2 km à La Moignerie, un peu en amont de Mordreuc,

- 4,8 km près de Plumazon, entre Mordreuc et Plouer,

- $7 \mathrm{~km}$ pour le point le plus aval, situé entre l'ancien et le nouveau pont de Port Saint-Jean/Port Saint-Hubert.
Les prélèvements sont effectués à trois profondeurs différentes (surface, milieu et fond) dans la colonne d'eau et le rejet est suivi pendant un cycle complet de marée, en morte-eau et en vive-eau. Le premier prélèvement a lieu avant le début du rejet de dragage, afin de connaître la turbidité ambiante, puis toutes les heures en chacun des points de surveillance jusqu'à la pleine mer du soir (fig. 2).

La turbidité est analysée soit par filtration (on obtient alors des valeurs de MES (en $\mathrm{mg} / \mathrm{l})$ ) soit par une méthode néphélométrique qui donne des valeurs en NTU (unité néphélométrique de turbidité), avec une relation entre ces deux paramètres de la forme: $\operatorname{MES}(\mathrm{mg} / \mathrm{l})=1,91 \mathrm{NTU}$ $-18,7$ et un coefficient de corrélation $r^{2}$ de 0,97 . Les résultats du suivi de rejet de dragage sont exprimés sous forme de diagrammes $3 \mathrm{D}$ où sont reportées, pour chaque épaisseur de la tranche d'eau, d'une part la distance du point de prélèvement par rapport à l'écluse du Chatelier, d'autre part en abscisse, les heures par rapport à la pleine mer et enfin en ordonnées les valeurs de turbidité ou de salinité des eaux.

\subsection{Résultats}

L'évolution de la salinité des eaux de surface au cours du suivi de rejet de dragage en marée de vive-eau (fig. 3) montre que les variations sont très ténues au niveau du Port Saint-Jean, la salinité passant de $33 \%$ à $30 \%$ entre la pleine mer et la basse mer. La partie comprise entre l'écluse du Chatelier et Mordreuc est celle qui connaît le plus de variations. Le point le plus amont a une salinité qui

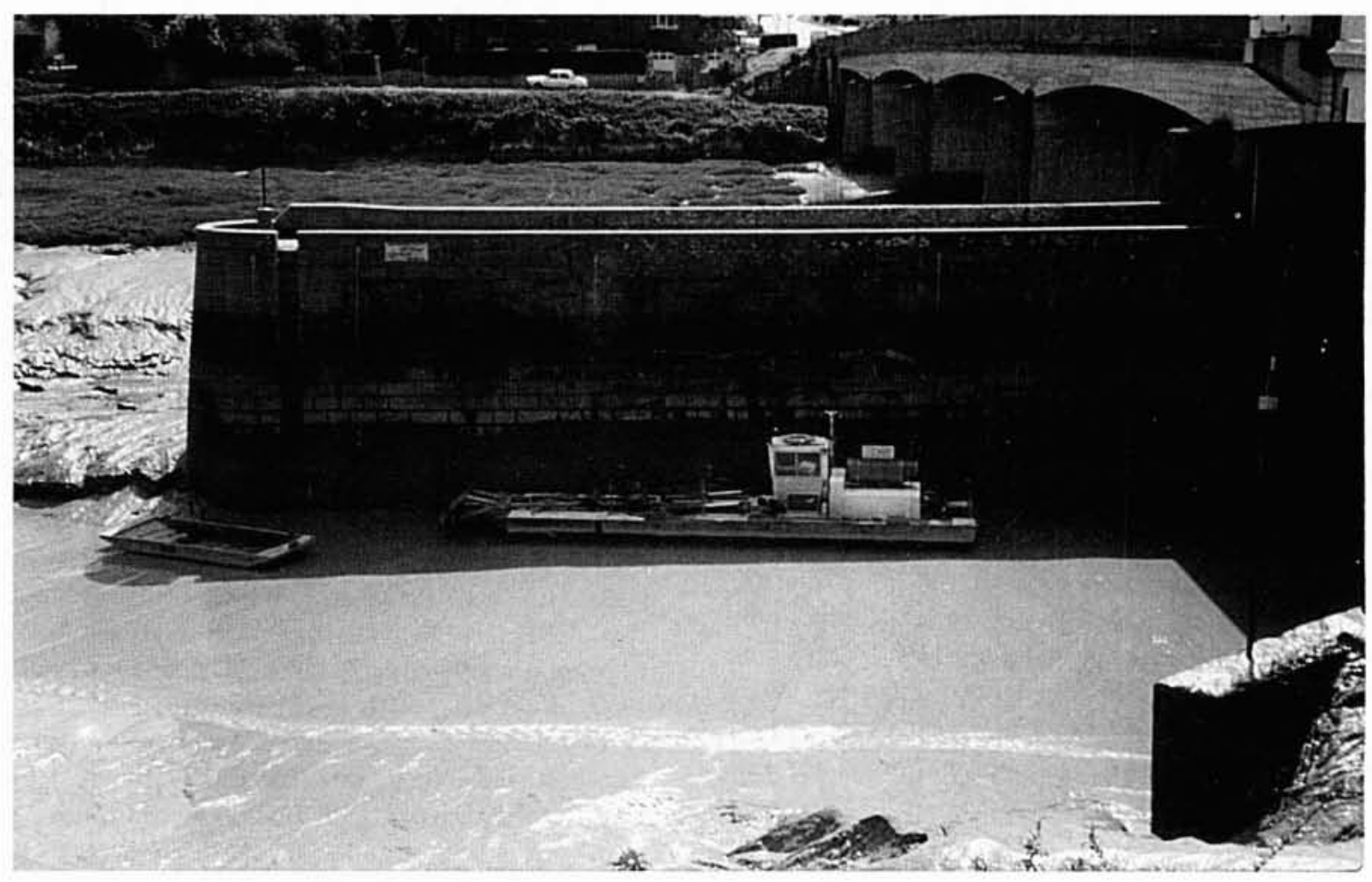

Photo $\mathbf{n}^{\circ} 1$ : Drague contre le musoir de l'écluse du Chatelier. 

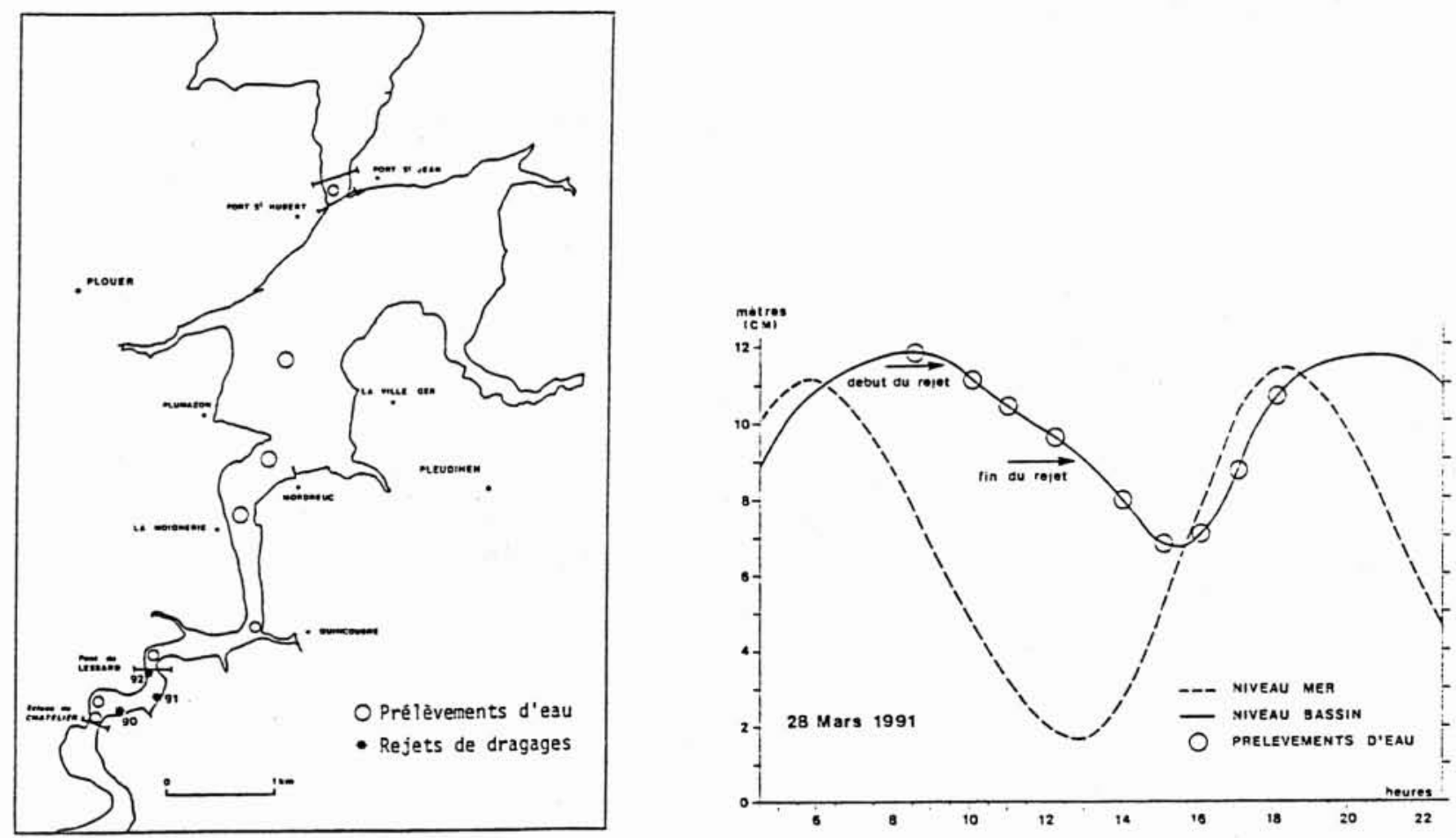

2. Localisation et chronologie des prélèvements d'eau pour le suivi de rejet de dragage (28 mars 1991).

passe de $15 \%$ à pleine mer à $3,9 \%$ à basse mer. Au niveau de Mordreuc, ces variations sont atténuées et la salinité en surface qui est de $24,4 \%$ à pleine mer chute à $13,7 \%$ à basse mer. La stratification haline entre les eaux de surface et les eaux de fond n'est bien marquée que dans la partie la plus amont de la zone estuarienne où, 2 heures avant la pleine mer, les eaux de surface ont une salinité comprise entre 5 et $15 \%$ tandis que les eaux de fond oscillent autour de $25 \%$ sur une partie de l'estuaire qui va jusqu'à $2 \mathrm{~km}$ à l'aval de l'écluse du Chatelier.

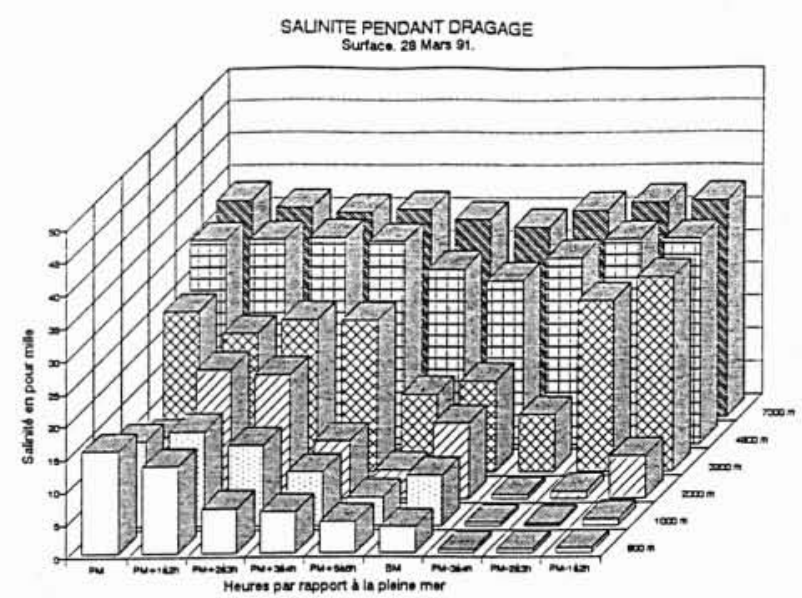

3. Evolution de la salinité en surface au cours du suivi du rejet de dragage en marée de vive-eau (28 mars 1991).
Par marée de vive-eau et avant le début du rejet de dragage, les teneurs en MES sont comprises entre 20 et $40 \mathrm{mg} / \mathrm{l}$ pour la partie amont de l'estuaire comprise jusqu'à $2 \mathrm{~km}$ en aval de l'écluse du Chatelier (fig.4). Vers Mordreuc, la turbidité chute entre 10 et $20 \mathrm{mg} / \mathrm{l}$ et est de l'ordre de $10 \mathrm{mg} / \mathrm{l}$ pour les points de Plouer et de Port Saint-Jean. Le début du rejet de dragage est très nettement marqué dans les eaux de fond des prélèvements amont qui passent à plus de $500 \mathrm{mg} / \mathrm{l}$ pour le point le plus proche du rejet et cette augmentation de turbidité se fait sentir jusqu'à $1 \mathrm{~km}$ en aval. Après une heure de rejet, la turbidité des eaux augmente considérablement à l'amont, jusqu'à $1,5 \mathrm{~g} / 1$ et, dans une moindre mesure, dans toute la partie étroite de l'estuaire comprise entre Le Chatelier et Mordreuc. Plus en aval, les teneurs en MES restent faibles, inférieures à $10 \mathrm{mg} / \mathrm{l}$ aussi bien à Plouer qu'au Port Saint-Jean (fig. 4).

Après la fin du rejet de dragage, à basse mer, les teneurs en MES de la partie amont sont élevées, supérieures à $100 \mathrm{mg} / \mathrm{l}$ et d'encore $60 \mathrm{mg} / \mathrm{l}$ peu avant Mordreuc. A Plouer et au Port Saint-Jean, les MES atteignent respectivement 25 et $15 \mathrm{mg} / \mathrm{l}$. Peu après la basse mer, les vannes de l'écluse du Chatelier ont été ouvertes ce qui a provoqué un violent courant de chasse et a permis le transport de grandes quantités de sédiment. En effet, les prélèvements effectués pendant cette chasse ont des teneurs en MES qui dépassent $3 \mathrm{~g} / \mathrm{l}$ à l'amont et $300 \mathrm{mg} / \mathrm{l}$ à Mordreuc. Le courant n'a été observé que pendant $1 / 2$ heure mais l'augmentation de la turbidité s'est poursuivie pendant au moins $2 \mathrm{~h} 30$ et à des niveaux très élevés puisque supérieurs à $1 \mathrm{~g} / \mathrm{l}$ dans les eaux de fond sur plus de $2 \mathrm{~km}$ à l'aval du Chatelier (fig. 4). 


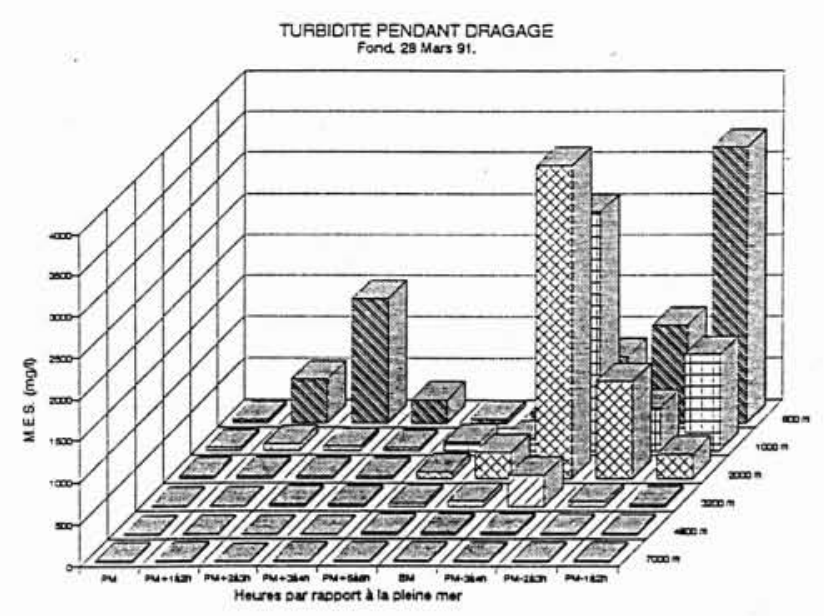

4. Evolution de la turbidité des eaux de fond entre l'écluse du Chatelier et Port Saint-Jean au cours de la marée de vive-eau du 28 mars 1993.

La remontée du flot empêche les matières en suspension de se disperser plus loin vers l'aval de l'estuaire, et la turbidité est faible à Plouer et à Port Saint-Jean et le restera jusqu'à la pleine mer. Au bout de 2 heures de flot, les MES se retrouvent concentrées à plus de $3 \mathrm{~g} / \mathrm{l}$ dans les eaux de fond les plus proches de l'écluse du Chatelier, et à 200$300 \mathrm{mg} / \mathrm{l}$ pour le point situé à $2 \mathrm{~km}$ de l'écluse. A Mordreuc, la turbidité se retrouve à un niveau de $20 \mathrm{mg} / \mathrm{l}$, un peu plus élevé qu'à la pleine mer précédente, mais 10 fois moins forte que pendant l'ouverture des vannes de l'écluse.

\section{Causes de l'accélération de l'envasement}

Lieu d'interférences entre les actions marines et les actions fluviatiles, le milieu estuarien est caractérisé par sa dynamique propre, dominée par la rythmicité des marées, mais fortement influencée par le débit du cours d'eau et les caractéristiques variables de celui-ci. D'aval en amont, l'onde de marée se propage avec un retard croissant et repousse les eaux douces apportées par le fleuve. Les eaux marines salées se mélangent aux eaux douces et une certaine stratification haline apparait, sous forme d'un gradient vertical dans les estuaires où l'action de la marée est prédominante comme dans le cas de la Rance.

Les gradients verticaux de densité donnent lieu à des courants résiduels, orientés généralement vers l'amont au fond et vers l'aval en surface (IPPEN et Harleman, 1969). A l'extrémité amont de l'intrusion saline se situe une zone de convergence des courants résiduels ou point nodal, qui suit les fluctuations saisonnières amont-aval de l'intrusion saline. Les matières en suspension piégées dans la zone du point nodal constituent le "bouchon vaseux » où la turbidité est très forte et dont la position moyenne dans l'estuaire dépend du débit fluvial (AlLEN et KLINGeBIEL, 1974). Le bouchon vaseux est repoussé en amont lors des marées de vive-eau et surtout en période d'étiage du cours d'eau. Au contraire, une crue fluviale pendant une période de morte-eau déplace le bouchon vaseux vers l'aval et peut même l'expulser au-delà de l'embouchure où il peut alimenter les vasières du plateau continental. Or le dépôt des sédiments en suspension est directement lié à la position du bouchon vaseux et varie donc en fonction des saisons.

En outre, même dans les zones à faible salinité, la concentration en sels est toujours suffisante pour permettre l'agglomération des particules sous forme d'agrégats dont la vitesse de chute est fortement augmentée. La turbulence du milieu interdit la plupart du temps la formation de ces agrégats et leur sédimentation sauf au moment des étales. Les matières en suspension forment alors une "crème de vase " près du fond (Photo $n^{\circ} 2$ ) et celle-ci ne sera reprise que si les forces mises en jeu au cours de la marée suivante suffisent pour vaincre la cohésion de ce matériel nouvellement déposé.

Les déplacements du bouchon vaseux se traduisent par une migration de la zone de sédimentation maximale, liée au calme des étales et notamment de l'étale de pleine mer dans la zone amont de l'estuaire. Dans la Rance, les mesures de turbidité ont montré que le bouchon vaseux était situé en 1991 très en amont dans le bassin maritime, à proximité de l'écluse du Chatelier où les teneurs en MES atteignent $100 \mathrm{mg} / \mathrm{l}$ en dehors des périodes de dragage, tandis que la turbidité naturelle n'excède pas $10 \mathrm{mg} / \mathrm{l}$ en aval de Mordreuc. Le débit fluvial de la Rance est très faible par rapport au volume du prisme tidal de telle sorte que la sédimentation préférentielle se trouve reportée à l'extrême amont de l'estuaire.

L'évolution naturelle d'un estuaire du type de la Rance se traduit donc par une accumulation des vases dans toutes les zones où l'agitation est faible et plus particulièrement à l'amont du bassin maritime, dans un chenal étroit en l'absence de clapots ou de chasse. Cette tendance naturelle à l'envasement n'explique cependant pas pourquoi le processus s'est accéléré depuis 1989 nécessitant des dragages d'entretien du chenal de navigation.

La position du bouchon vaseux étant directement liée à l'équilibre entre le débit fluvial et la marée, il est nécessaire de déterminer si des changements sont intervenus dans les débits de la Rance. A l'amont, la Rance fluviale est barrée au niveau de Rophémel et les débits sortants de ce barrage constituent une bonne estimation des débits de la Rance. L'évolution des débits sortants à Rophémel depuis 1975 (fig. 5) montre que quatre années, 1976, 1989, 1991 et 1992 se caractérisent par de très faibles débits, inférieurs à $2 \mathrm{~m}^{3} / \mathrm{s}$. Les autres années ont des débits supérieurs à $4 \mathrm{~m}^{3} / \mathrm{s}$ pour certains mois, et le plus souvent compris entre 6 et $10 \mathrm{~m}^{3} / \mathrm{s}$. Quatre années, 1981, 1982, 1983 et $1988 \mathrm{se}$ distinguent par des mois à forts débits, de 12 à $14 \mathrm{~m}^{3} / \mathrm{s}$.

Ce diagramme (fig. 5) montre clairement que la Rance a connu un étiage prononcé en 1989, 1991 et 1992. L'année 1976, à faible débit, a été suivie en 1977 par des débits importants pendant plusieurs mois. A l'inverse, les débits de 1989, très déficitaires, n'ont été suivis en 1990 que par un mois à débit de l'ordre de $9 \mathrm{~m}^{3} / \mathrm{s}$, et trois autres mois à très faible débit d'environ $1 \mathrm{~m}^{3} / \mathrm{s}$. Le débit de la Rance a donc été à son niveau le plus bas pendant presque quatre 


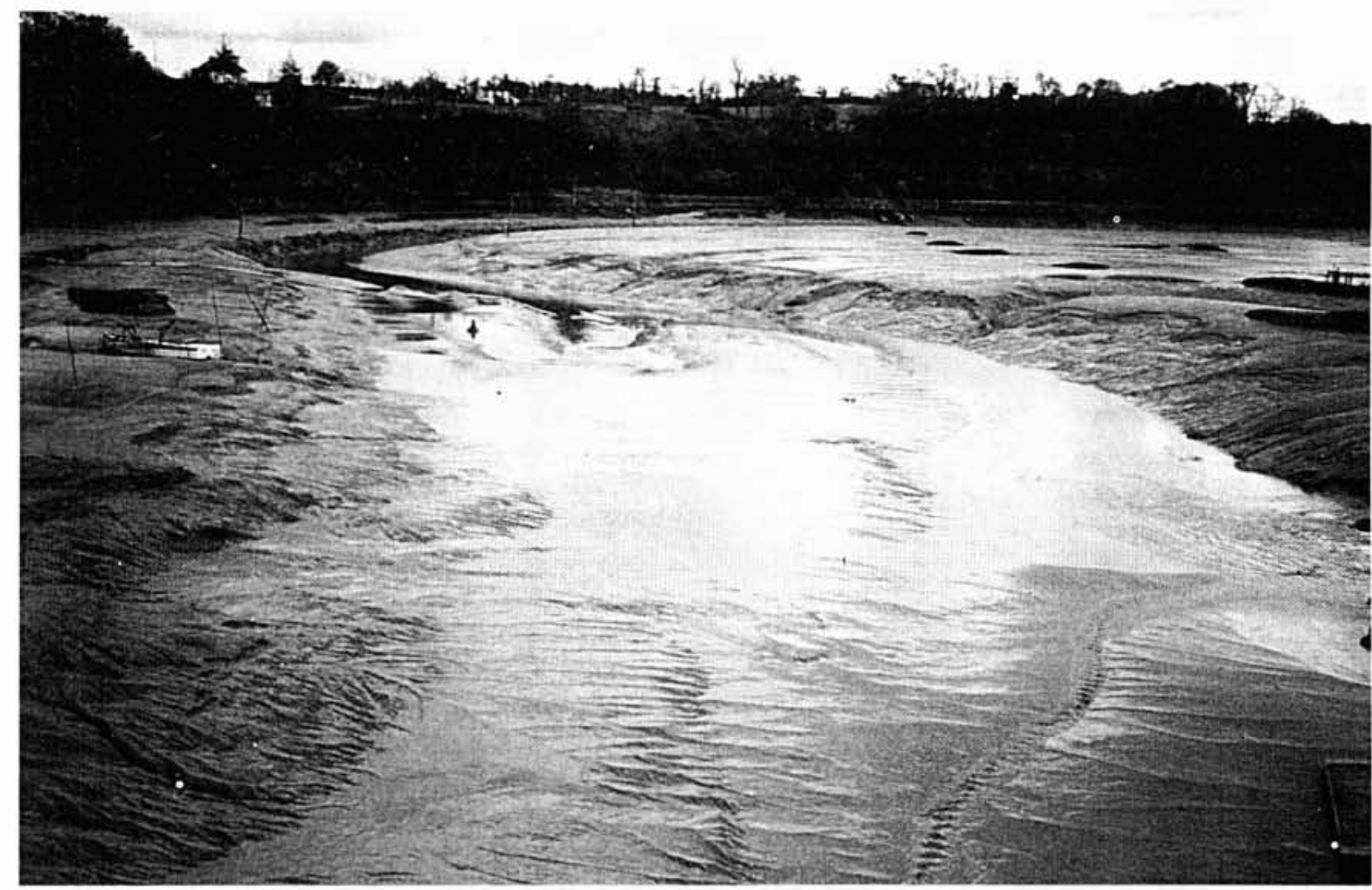

Photo $\mathbf{n}^{\circ} 2$ : Dépôt de la crème de vase à l'amont de l'estuaire.

années consécutives, et c'est précisément depuis cette période que les problèmes d'envasement se sont accrus.

Ces observations sur l'évolution des débits de la Rance peuvent être corrélées au suivi de la pluviométrie dans le bassin versant depuis 20 ans (fig. 6). Cinq stations réparties sur l'ensemble du bassin versant de la Rance ont été retenues et sont, d'amont en aval :

- Caulnes, en rive gauche de la Rance, à l'amont du barrage de Rophémel ;

- Saint-Pern, en rive droite, à $8 \mathrm{~km}$ à l'Est de Rophémel ;

- Lanvallay, en rive droite, à $2 \mathrm{~km}$ en amont de Dinan :

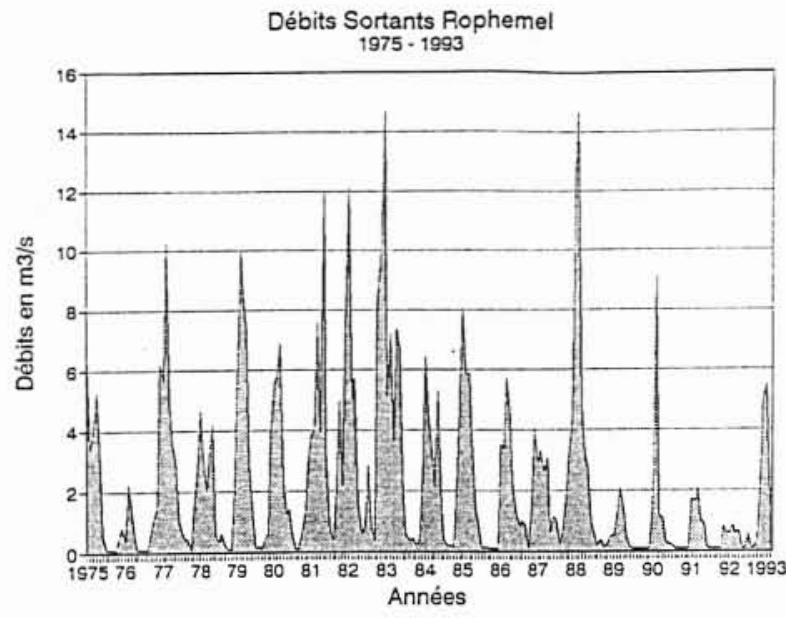

5. Evolution des débits sortants à Rophèmel depuis 1975 .
- Plesder, en rive droite de la Rance, à $10 \mathrm{~km}$ dans le Sud-Est de Dinan ;

- Dinard-Pleurtuit, en rive gauche, pour la station la plus aval de l'estuaire.

A l'amont du bassin versant, pour la station de Caulnes, la moyenne annuelle 1971-1990 des précipitations est de $694 \mathrm{~mm}$. Les années 1989, 1990 et 1991 ont été déficitaires de $22 \%, 17 \%$ et $9 \%$ respectivement par rapport à la moyenne annuelle, tandis que les années 1981 et 1982 ont au contraire eu une pluviométrie supérieure de près de $30 \%$ par rapport à cette moyenne. A Saint-Pern, la

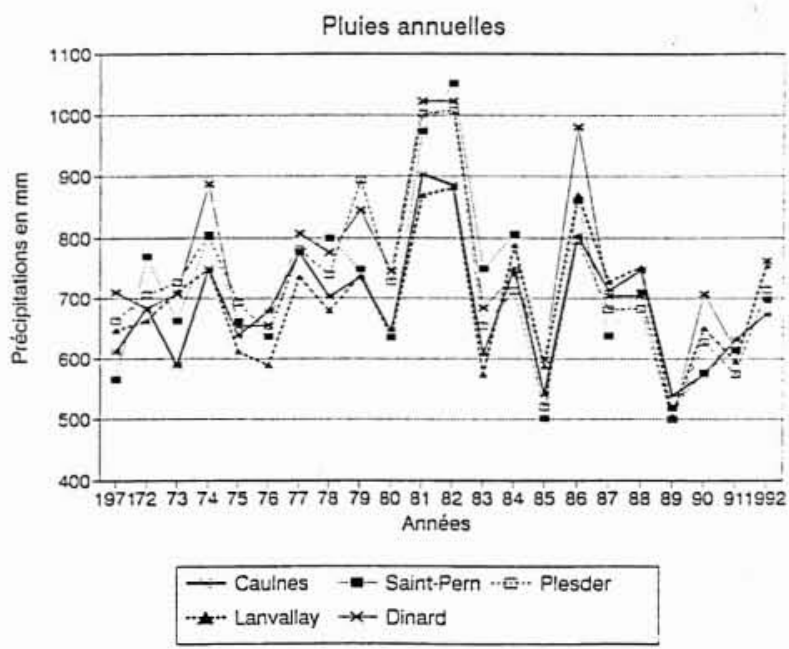

6. Pluviométrie dans le bassin versant de la Rance pour la période 1971-1992. 


\section{BONNOT-COURTOIS}

moyenne des précipitations entre 1971 et 1990 est de $712 \mathrm{~mm}$. Les années 1981 et 1982, toujours pluvieuses, dépassent de $36 \%$ à $47 \%$ cette moyenne, alors que 1989 est déficitaire de $27 \%, 1990$ de $19 \%$ et 1991 de $14 \%$.

A Lanvallay, pour une moyenne 1971-1990 de $697 \mathrm{~mm}$, le déficit pluviométrique atteint $27 \%$ en $1989,7 \%$ en 1990 et $15 \%$ en 1991. A Plesder, pour une moyenne de $727 \mathrm{~mm}$, on observe le même déficit de $31 \%$ en 1989 , de $14 \%$ en 1990 et de $21 \%$ en 1991, avec toujours des années 1981 et 1982 très pluvieuses, dépassant la moyenne de $38 \%$. Tout à fait à l'aval du bassin versant, la moyenne des précipitations enregistrées à Dinard a été de $756 \mathrm{~mm}$ entre 1971 et 1990. L'année 1989, très sèche présente un déficit de $34 \%$ par rapport à cette moyenne, et les années 1990 et 1991 de $8 \%$ et $19 \%$ respectivement.

Du point de vue pluviométrie, les années 1989, 1990 et 1991 sont donc nettement déficitaires par rapport à la moyenne, quelle que soit la station du bassin versant considérée. L'ensemble du bassin versant a eu une pluviométrie inférieure à la moyenne de $28 \%$ en 1989 , de $13 \%$ en 1990 et de $15,5 \%$ en 1991. Ces trois années consécutives de sécheresse ont provoqué une réduction importante des débits de la Rance. En conséquence, la forte diminution des apports fluviatiles au niveau de l'écluse du Chatelier a favorisé les conditions d'une remontée du bouchon vaseux et de la zone de sédimentation préférentielle vers l'amont du bassin maritime. La sécheresse et donc l'étiage prolongé de la Rance constituent la principale cause de l'accélération de l'envasement depuis 1990.

L'année 1992 a été marquée par une amélioration de la pluviométrie qui approche, voire dépasse la moyenne annuelle dans toutes les stations du bassin versant. La situation de l'envasement s'est également améliorée puisqu'il n'a pas été nécessaire de procéder à un dragage d'entretien du chenal au début de l'année 1993. Par contre, l'expérience de la chasse accidentelle rencontrée au cours du suivi de rejet de dragage réalisé en 1991 a conduit à proposer la réalisation d'une chasse hydraulique expérimentale afin de suivre son efficacité sur la position du bouchon vaseux et donc sur les possibilités d'évacuer la crème de vase sédimentée contre l'écluse du Chatelier vers l'aval du bassin maritime.

\section{Suivi d'une chasse hydraulique expérimentale}

Les grandes quantités de sédiments remis en suspension et l'extension vers l'aval dủ panache turbide observées au cours de la chasse ayant eu lieu pendant le suivi des rejets de dragage permettaient d'envisager d'utiliser la masse d'eau retenue en arrière de l'écluse du Chatelier comme potentiel de chasse hydraulique afin d'évacuer vers l'aval les vases du chenal de navigation. A l'automne 1992 (fig.6), les conditions pluviométriques ayant été plus favorables que les années précédentes, il a ainsi été possible de retenir un certain volume d'eau dans le bief amont compris entre les écluses du Chatelier et de Léhon et de réaliser des chasses hydrauliques. La dynamique des matières en suspension a été suivie au cours de ces chasses expérimentales, en février 1993, afin de connaître les quantités de vases remises en suspension et l'extension vers l'aval du panache turbide en fonction des conditions hydrologiques du bassin.

\subsection{Conditions expérimentales}

La répartition des points de prélèvements est la même que celle des prélèvements effectués en 1991 (BONNOTCourtors et al., 1993) pour le suivi des rejets de dragage, mais un certain nombre de points intermédiaires y ont été ajoutés (fig. 7) afin d'avoir un profil détaillé de l'extension du panache turbide dans toute la partie amont du bassin maritime. Les opérations de chasse ont débuté 1 h 30 avant le bas niveau du bassin maritime et se sont poursuivies pendant une heure (fig. 7), avec un débit de $10 \mathrm{~m}^{3} / \mathrm{s}$ la première demi-heure et de $42 \mathrm{~m}^{3} / \mathrm{s}$ la deuxième demi-heure (Photo $n^{\circ} 3$ ). La fin de la chasse expérimentale a eu lieu $1 / 2$ heure avant la basse mer et le volume total d'eau douce relâché a été de l'ordre de $93000 \mathrm{~m}^{3}$. Les courants mesurés atteignent $1,5 \mathrm{~m} / \mathrm{s}$ à $300 \mathrm{~m}$ en aval de l'écluse du Chatelier et se maintiennent supérieurs à $1 \mathrm{~m} / \mathrm{s}$ jusqu'à environ $1 \mathrm{~km}$ en aval puis ils s'amortissent jusqu'à $0,7 \mathrm{~m} / \mathrm{s}$ à $2 \mathrm{~km}$ en aval de l'écluse. L'étude de la viscosité et de la rigidité initiale de la crème de vase avait permis de déterminer la vitesse critique d'érosion des sédiments de surface non consolidés et aboutissait à des vitesses de courant de l'ordre de $1,15 \mathrm{~m} / \mathrm{s}$ à $1 \mathrm{~m}$ du fond. Cette valeur est inférieure à celle mesurée au cours de la chasse expérimentale, et les débits ont donc été suffisants pour provoquer l'érosion de la couche superficielle de vase molle près du musoir de l'écluse du Chatelier. Mais, à partir de $30 \mathrm{~cm}$ de profondeur dans le sédiment, les valeurs de cohésion mesurées in situ montrent que les vases sont consolidées et leur érosion nécessite des vitesses de courants beaucoup plus élevées. Les prélèvements ont été effectués avant le début de la chasse, puis à basse mer et au cours de la remontée du flot jusqu'à la pleine mer suivante. La relation entre les teneurs en MES en mg/l et les valeurs néphélométriques de turbidité est de la forme : MES mg/l=1,36 NTU - 19,9 avec un coefficient de corrélation $r^{2}$ de 0,97 .

\subsection{Résultats}

Avant le début de la chasse (fig. 8), les eaux saumâtres sont comprises dans la partie étroite de l'estuaire comprise entre Mordreuc et Le Chatelier. La salinité des eaux de surface est de $20 \%$ et celle des eaux de fond de 25 à $30 \%$ pour les points situés à plus de $2 \mathrm{~km}$ de l'écluse du Chatelier. Une nette stratification haline apparaît à l'amont et jusqu'à $1 \mathrm{~km}$ de l'écluse, les eaux de surface ayant une salinité de $5 \%$ et les eaux de fond de 20 à $25 \%$.

Au moment de la chasse, un profil intermédiaire réalisé dans la partie amont de l'estuaire montre que la salinité est très faible sur toute l'épaisseur de la tranche d'eau, les eaux douces s'étendant vers l'aval jusqu'à au moins $2 \mathrm{~km}$ de l'écluse du Chatelier. Une heure après la fin de la chasse, le front de salinité est repoussé jusqu'à plus de $2 \mathrm{~km}$ en aval 

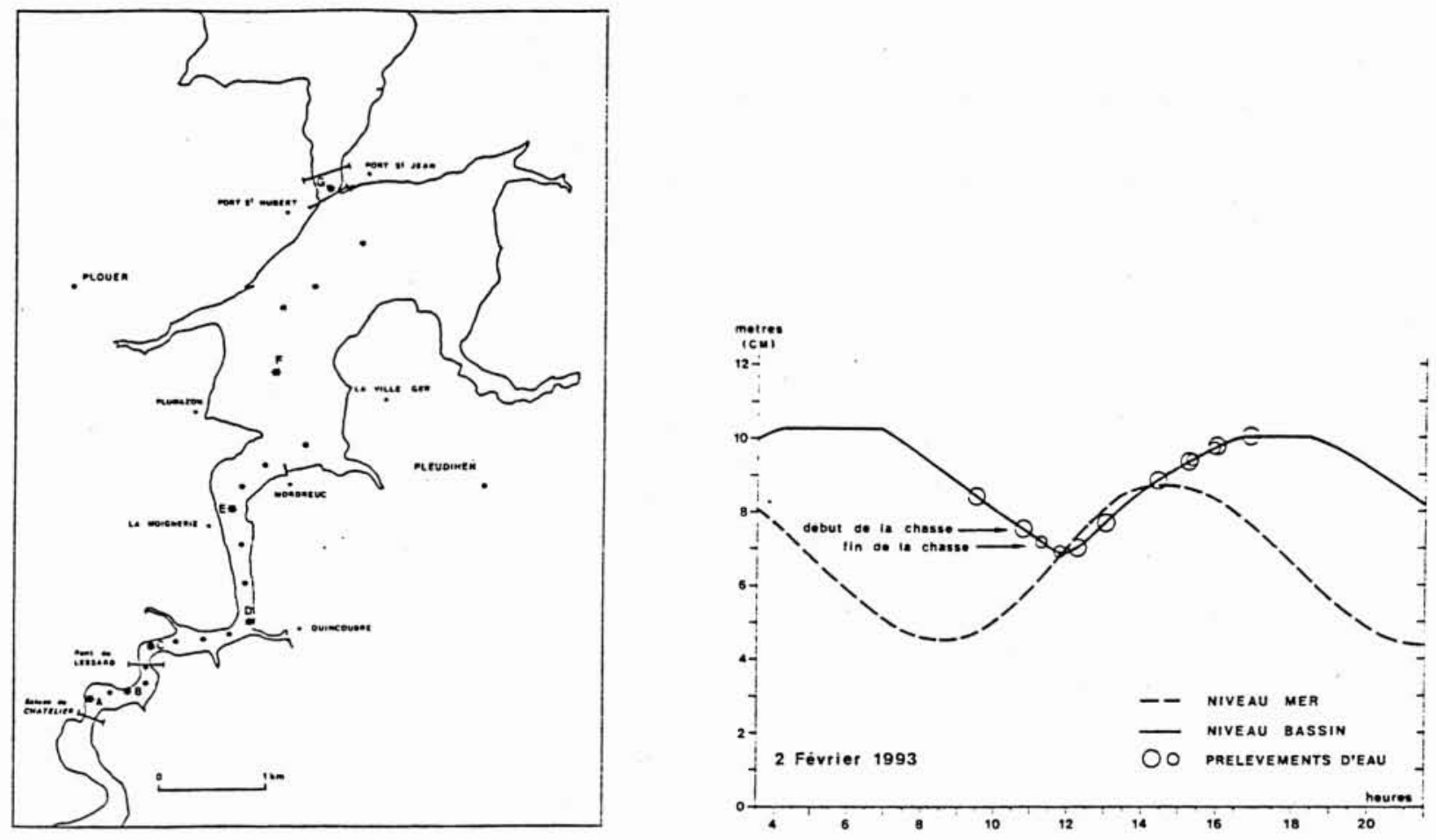

7. Localisation et chronologie des prélèvements au cours de la chasse hydraulique expérimentale (2 février 1993).

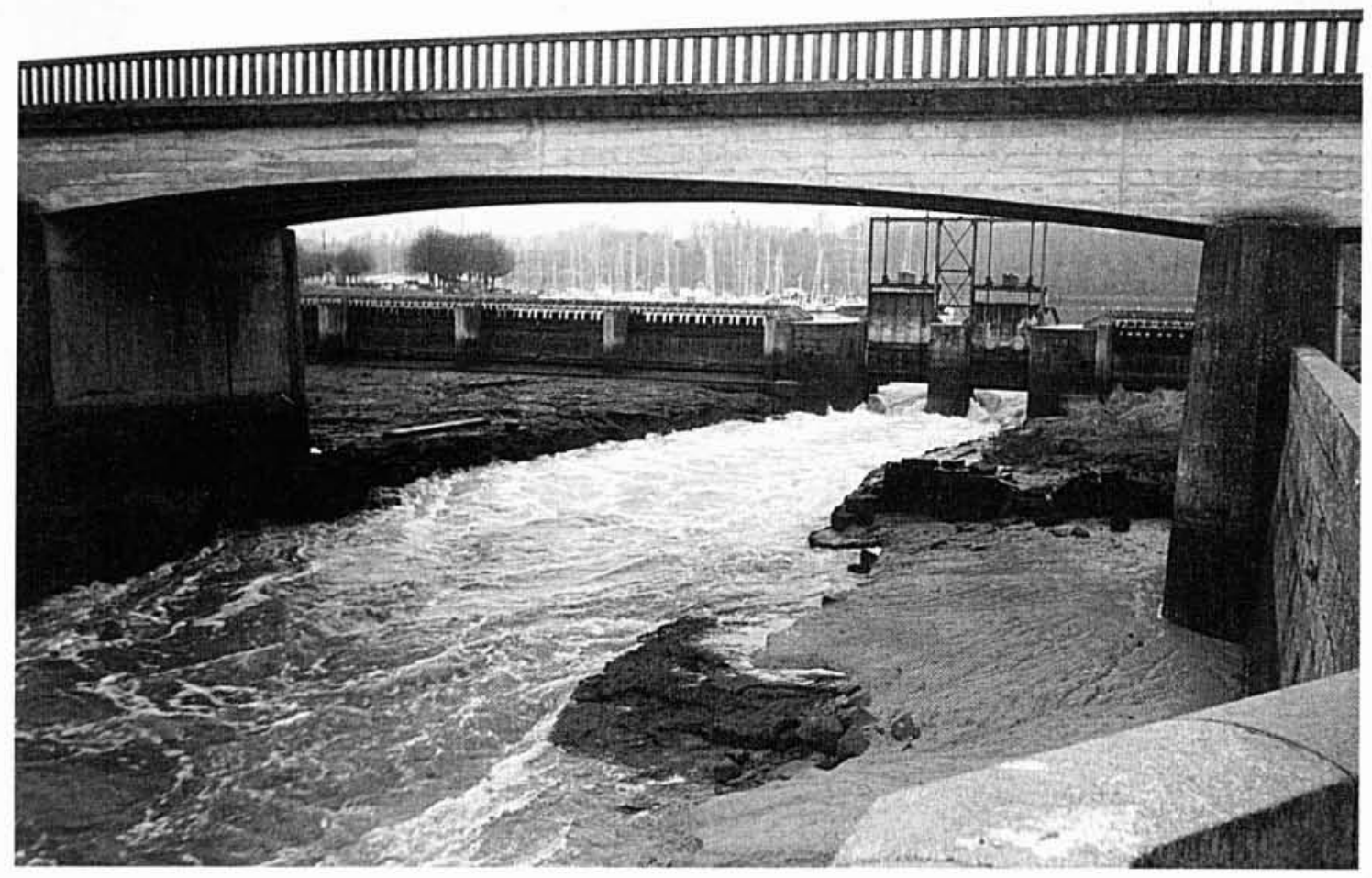

Photo $\mathbf{n}^{\circ} 3$ : Chasse hydraulique expérimentale par les vannes de l'écluse du Chatelier.

de l'écluse où les eaux sont douces sur toute l'épaisseur de la tranche d'eau. Les salinités deviennent ensuite intermédiaires $(10$ à $15 \%$ ) jusqu'à Mordreuc, puis, au-delà, les eaux restent salées jusqu'au Port Saint-Jean.
L'arrivée du flot dans la partie amont de l'estuaire se traduit par une remontée du front de salinité jusqu'à $2 \mathrm{~km}$ de l'écluse à ce moment de la marée, soit 3 heures après la basse mer. Un peu plus tard, un profil intermédiaire 
C. BONNOT-COURTOIS
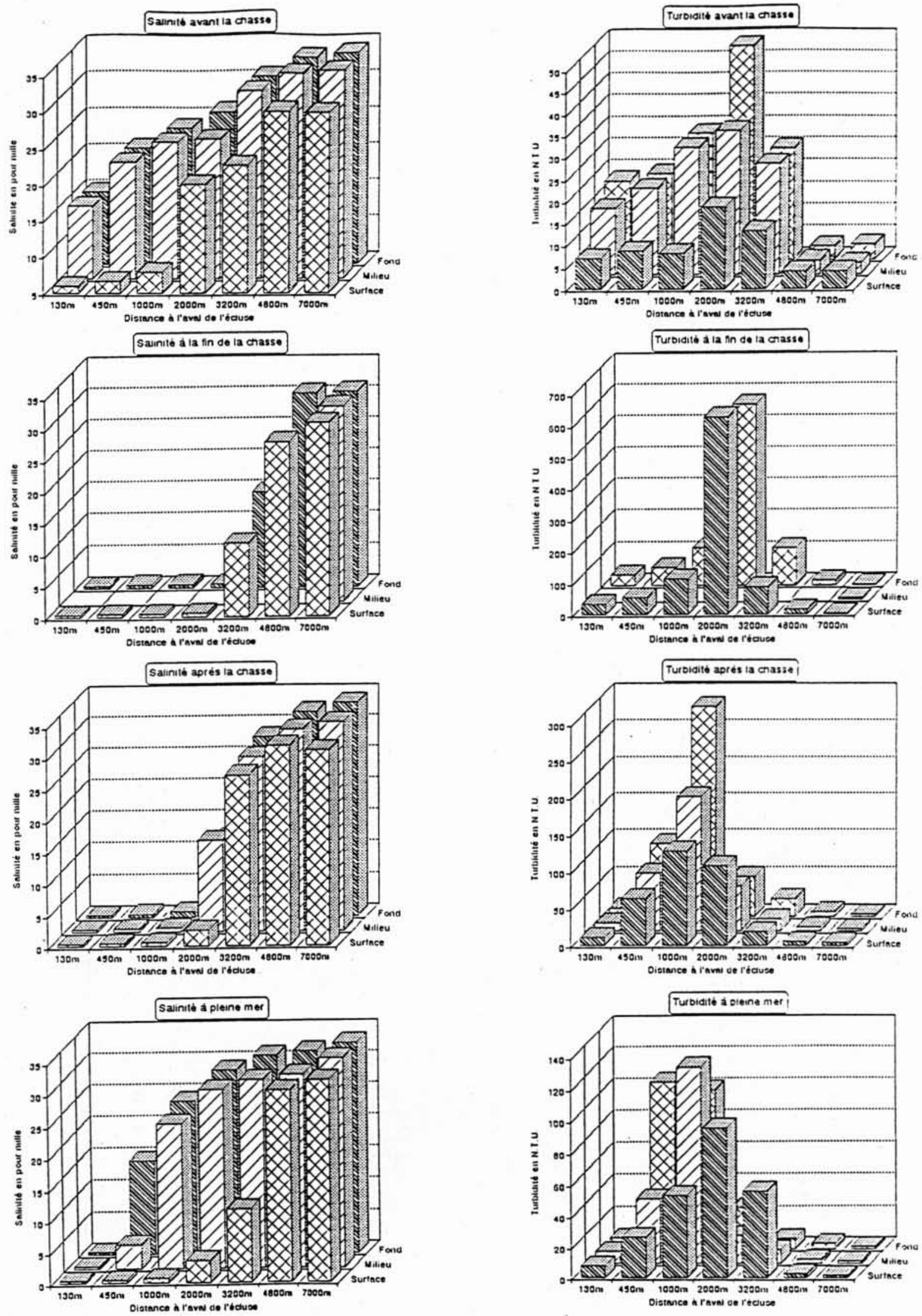

8. Répartition de la salinité au cours de la chasse hydraulique expérimentale.

9. Répartition de la turbidité au cours de la chasse hydraulique expérimentale. 
montre une stratification haline très marquée jusqu'au Pont de Lessard ( $800 \mathrm{~m}$ en aval du Chatelier) où les eaux de surface sont pratiquement des eaux douces alors qu'au fond, la salinité varie de 10 à $25 \%$.

La pleine mer suivant la chasse hyraulique provoque une remontée des eaux salées de fond jusqu'à $450 \mathrm{~m}$ de l'écluse du Chatelier (fig. 8). Les eaux de surface comprises entre Port Saint-Jean et Mordreuc restent salées alors que l'influence des eaux douces se fait sentir vers l'aval à plus de $3 \mathrm{~km}$ de l'écluse du Chatelier.

Avant la chasse, la turbidité entre Port Saint-Jean et Mordreuc est très faible de l'ordre de 5 NTU (fig. 9). Les teneurs en MES augmentent quand on remonte vers l'amont, avec un maximum de turbidité à $2 \mathrm{~km}$ en aval (45 NTU pour les eaux de fond) de l'écluse du Chatelier, puis la turbidité diminue de nouveau jusqu'à $15 \mathrm{NTU}$ à proximité de l'écluse. Au début de la chasse, les turbidités augmentent brutalement jusqu'à 800 NTU dans les eaux de fond près de l'écluse, puis, pendant la chasse, les valeurs de turbidité augmentent d'un facteur $10 \mathrm{en}$ aval du Pont de Lessard (600 NTU à $800 \mathrm{~m}$ et près de 1000 NTU à $2 \mathrm{~km}$ en aval de l'écluse).

Une heure après la fin de la chasse, le maximum de turbidité se situe toujours au niveau du point D (600 NTU), tandis que de part et d'autre de ce point, entre Le Chatelier et Mordreuc, les teneurs en MES chutent à quelques dizaines de NTU. A l'aval de Mordreuc, les turbidités restent faibles à $5 \mathrm{~km}$ de l'écluse $(15 \mathrm{NTU})$ et plus encore au Port Saint-Jean (3 NTU).

Après la chasse, la concentration des matières en suspension atteint son maximum entre 1 et $2 \mathrm{~km}$ en aval de l'écluse (270 NTU pour les eaux de fond). La remontée du flot repousse les matières en suspension vers la partie amont de l'estuaire, particulièrement entre Mordreuc et Le Chatelier. A la pleine mer suivante (fig. 9), le bouchon vaseux est de nouveau centré à l'aval du pont de Lessard.

\section{Conclusion}

L'étroitesse du chenal de navigation entre Mordreuc et l'écluse du Chatelier interdit le passage d'engins de dragage trop importants et leur puissance est de ce fait limitée. La localisation des points de rejets était très proche de l'écluse du Chatelier, à environ $1000 \mathrm{~m}$, et la turbidité induite par le rejet était de plus de $500 \mathrm{mg} / \mathrm{l}$ dans les eaux de fond au début de rejet et de plus de $1,5 \mathrm{~g} / 1$ près du rejet après une heure de dragage. Même si le rejet n'a lieu que pendant la vidange du bassin, le suivi du panache turbide a montré que son extension vers l'aval était limitée à la zone amont de Mordreuc, la turbidité n'ayant que très faiblement augmenté en aval de Mordreuc jusqu'au Port Saint-Jean. Les seuls courants de jusant sont donc insuffisants pour transporter les matières en suspension très loin en aval. En effet, en l'absence de chasse efficace, le panache turbide du rejet de dragage et le bouchon vaseux naturel de la Rance restent bloqués à l'amont où ils se déposent sous forme de crème de vase, dont l'évolution du niveau a été suivie à l'écluse du Chatelier après les opérations de dragage.
En 1991, la hauteur de la vase atteinte à la fin des opérations de dragage est restée à peu près stable pendant 2 mois, avec une augmentation du niveau de $10 \mathrm{~cm}$. Puis, pendant la période estivale, la hauteur de vase a progressé d'environ $8 \mathrm{~cm}$ par semaine pendant près de 3 mois. A partir du mois d'octobre, la situation s'est alors stabilisée à un niveau de vase supérieur d'l $\mathrm{m}$ à celui qui existait à la fin du dragage. Une grande partie de la vase est donc revenue sédimenter contre l'écluse du Chatelier, puisque l'on a une accumulation de $1 \mathrm{~m}$ d'épaisseur en 4 mois dans l'écluse. La remontée du niveau a été spectaculaire pendant les mois d'été, caractérisés par une quasi-absence de pluies tandis que le phénomène s'est ralenti dès le mois d'octobre. Si le dragage d'entretien du chenal de navigation est efficace à court terme, son impact à moyen ou long terme est plus discutable puisque la sédimentation a repris inexorablement ses droits à proximité de l'écluse du Chatelier en raison de la remontée du bouchon vaseux liée au très faible débit fluvial de la Rance, conséquence d'une longue période de sécheresse.

Les pluies survenues à la fin de l'année 1992 ont permis de rétablir des débits à Rophémel plus soutenus et donc d'alimenter l'amont de l'estuaire. En outre, ont été effectuées un certain nombre de chasses, soit demandées à titre expérimental pour dégager le chenal de navigation, soit pour la régulation du plan d'eau à l'amont. Ces chasses ont eu pour effet de rétablir l'équilibre entre le débit de la Rance et l'action de la marée et le bouchon vaseux s'est déplacé vers l'aval jusqu'à environ $3 \mathrm{~km}$ de l'écluse. Les suivis de chasse expérimentale ont donc été réalisés dans une situation d'envasement beaucoup moins critique qu'au cours des années précédentes puisque le bouchon vaseux et la crème de vase molle déposée dans la partie amont du chenal de navigation en période de sécheresse avaient déjà été déplacés à quelques $\mathrm{km}$ vers l'aval.

Les chasses expérimentales ont été réalisées environ une heure avant la basse mer, avec un débit fluvial de l'ordre de $40 \mathrm{~m}^{3} / \mathrm{s}$ et des niveaux du bassin maritime correspondant à des marées de faible coefficient. Dans ces conditions, le panache turbide s'est étendu vers l'aval jusqu'au niveau de Mordreuc, à $4 \mathrm{~km}$ de l'écluse du Chatelier. Les courants à proximité de l'écluse du Chatelier étaient de l'ordre de $1,5 \mathrm{~m} / \mathrm{s}$ mais ils s'amortissent assez rapidement à $2 \mathrm{~km}$ en aval jusqu'au niveau à partir duquel on observe un premier élargissement du chenal jusqu'à Mordreuc où la vitesse des courants tombe en dessous de $0,5 \mathrm{~m} / \mathrm{s}$. Cette atténuation des courants est encore plus marquée à l'aval de Mordreuc et de la pointe du Chêne Vert où le chenal s'élargit dans la plaine de Mordreuc jusqu'au Port SaintJean. Le front de turbidité est directement lié au front de salinité de la dynamique des matières en suspension suit les lois et la dynamique estuarienne résultant de l'équilibre entre les débits d'eau douce et le prisme de marée.

Quantitativement, les plus fortes turbidités s'observent tout au début des chasses à proximité de l'écluse du Chatelier. Les teneurs en MES sont de l'ordre de $1,5 \mathrm{~g} / 1$ pour un débit de $40 \mathrm{~m}^{3} / \mathrm{s}$. Une fois le courant de chasse établi, la turbidité à l'amont diminue un peu et se stabilise à un peu moins d'l g/l. Au niveau du bouchon vaseux, la turbidité 


\section{BONNOT-COURTOIS}

passe de quelques mg/l avant la chasse à plus de $1 \mathrm{~g} / \mathrm{l}$ au début de la chasse. Les valeurs sont de l'ordre de $800 \mathrm{mg} / \mathrm{l}$ une heure après la fin de la chasse, puis diminuent à $110 \mathrm{mg} / \mathrm{l}$ quatre heures après la chasse pour tomber à $100 \mathrm{mg} / \mathrm{l}$ à la pleine mer suivante.

Ces valeurs soulignent le fait que l'efficacité de la remise en suspension est la plus grande au début de la chasse. Pour un volume d'eau amont donné, il est plus efficace de réaliser des chasses nombreuses mais de courte durée plutôt que de prolonger la durée des chasses surtout avec un débit moyen. En outre, en période de sécheresse, une chasse de courte durée réclamera moins d'eau, et ce au moment où son action sera la plus nécessaire. Les conditions optimales dans lesquelles ces chasses doivent être réalisées doivent permettre:

- de remettre les sédiments en suspension;

- d'assurer le transport des matières en suspension suffisamment loin vers l'aval et en un temps assez court avant que la remontée du flot ne retransporte les suspensions vers l'amont ;

- d'éventuellement laisser aux vases la possibilité de décanter à basse mer dans les parties les plus en aval de l'estuaire.

Que ce soit par la méthode des dragages limités ou par l'utilisation de chasses plus effectives, le problème du recyclage des sédiments remis en suspension et de leur retour dans les zones de sédimentation préférentielle de l'amont de l'estuaire reste posé. La rapide remontée des fonds au droit de l'écluse après les dragages de 1991 souligne l'importance de ce recyclage qui atteint une grande ampleur tant que les conditions de sécheresse se maintiennent. Ce phénomène n'est qu'une manifestation ordinaire et prévisible de la dynamique sédimentaire estuarienne, dont l'intensité peut être accrue du fait de la prolongation artificielle des étales et de la diminution des marnages réels. Les seuls courants de jusant sont trop faibles pour que le nuage turbide issu des sédiments dragués puisse quitter la zone étroite de l'amont de Mordreuc et s'étaler dans les secteurs plus élargis qui s'étendent plus au Nord.

Bien qu'aucune chasse hydraulique ne puisse probablement rejeter les sédiments en aval de l'étroiture du Port Saint-Jean, et a fortiori en dehors du bassin maritime, il apparaît comme plus efficace de disperser aussi complètement que possible vers l'aval les suspensions afin de permettre un dépôt de ces sédiments dans la zone élargie de la plaine de Mordreuc et d'obtenir un retour limité de ces matériaux dans le chenal de navigation lors du flot suivant. Une telle politique de rejet dans le lit de la Rance implique le transfert d'une partie de l'envasement du Chatelier vers les zones situées plus en aval qui, en dehors du chenal proprement dit, demeurent très réceptives aux phénomènes d'atterrissement préférentiel des sédiments. En cas de sécheresse, la mise en œuvre des chasses expérimentales nécessite par ailleurs une coordination dans la gestion des plans d'eau afin de pouvoir réserver le peu d'eau qui arrive dans le bassin versant et la relâcher ponctuellement pour évacuer les vases qui s'accumulent à l'amont de l'estuaire de la Rance.

\section{Références bibliographiques}

Allen G.P. et Klingebiel A. (1974). - La sédimentation estuarienne : exemple de la Gironde. Bull. Centre Rech. Pau. $S N P A$, v. $8, \mathrm{n}^{\circ} 1$, p. 263-293.

Berthois L. et C. (1954). - Etude de la sédimentation dans l'estuaire de la Rance. Première partie : Granulométrie des sédiments. Bull. Lab. Maritime de Dinard, fasc. XL, p. 414.

Berthois L. et C. (1955). - Etude de la sédimentation dans l'estuaire de la rance. Deuxième partie: Minéralogie des sédiments. Bull. Lab. Maritime de Dinard, fasc. 41, p. 3-18.

BONNOT-Courtois C. et LAFOND L.R. (1991). - Caractérisation et comportement des vases dans l'estuaire de la Rance. Rapport E.D.F./RIVAGES/Lab. Géomorphologie Dinard, $114 \mathrm{p}$.

Bonnot-Courtois C., Lafond L.R. et Le Vot M. (1993), Suivi d'une chasse hydraulique expérimentale dans la partie amont du bassin maritime de la Rance. Rapport E.D.F./RIVAGES/Lab. Géomorphologie Dinard, 23 p.

Bourcart J. et Roa Morales P. (1957). - Les dépôts vaseux de la Rance maritime et du Mont Saint-Michel. Bull. Soc. Géol. Fr. (6), VII, p. 545-552.

B.R.G.M. (1964). - Carte géologique au $1 / 80000^{\circ}$. Feuille de Dinan. $3^{\mathrm{e}}$ édition.

Choux J. (1959). - Contribution à l'étude sédimentologique des formations meubles de la Rance maritime. Bull. Soc. Géol. Min. Bretagne, fasc. 1, p. 37-66.

FraLiT (1977). - Télédétection du littoral océanique de la France. Collection E.N.S. Montrouge, $\mathrm{n}^{\circ} 11,310 \mathrm{p}$.

IPPEN A.T. et Harleman M. (1969). - Salinity intrusion effects in estuary choaling. Proc. Amer. Soc. Civ. Engin., 95, p. 9-27.

L.C.H.F. (1982), - Etude sédimentologique de l'estuaire de la Rance. Rapport Général Institution interdépartementale pour l'aménagement de la Rance propre. DDE Ille-etVilaine, $21 \mathrm{p}$.

L.C.H.F. (1986). - Evolution des conditions de navigation dans la partie amont de l'estuaire de la Rance. Rapport EDF, CRTT Ouest, GME Bretagne/DDE Ille-et-Vilaine, $15 \mathrm{p}$.

RETIÉRE C. (1989), - Energie marémotrice et environnement aquatique. La Houille Blanche, $n^{\circ} 2$, p. 133-147.

Retière C. et Richard O. (1980). - Conséquences écologiques de 10 années de fonctionnement de l'usine marémotrice de la Rance. Rapport EDF/Lab. Mar. Dinard, 71 p.

Roa Morales P. (1956). - La marée de salinité, la turbidité et les sédiments de la Rance maritime. Thèse Dr. Univ. Paris, $157 \mathrm{p}$. 